\title{
CLINICAL CORRELATION OF DOPPLER VELOCIMETRY AND INTRAUTERINE GROWTH RESTRICTION (IUGR)
}

\author{
Jagadish G. Sutagatti ${ }^{1}$, S. K. Joshi² \\ ${ }^{1}$ Associate Professor, Department of Radiodiagnosis, Karnataka Institute of Medical Sciences (KIMS), Hubli. \\ ${ }^{2}$ Professor and HOD, Department of Radiodiagnosis, SDM College of Medical Sciences and Hospital, Dharwad.
}

\section{ABSTRACT}

\section{BACKGROUND}

Doppler velocimetry is the only non-invasive method for assessing the foetoplacental haemodynamic status. We conducted a study to find out the efficacy of Doppler study as a screening test for Intrauterine Growth Restriction (IUGR) and to find the best Doppler index for this purpose.

\section{MATERIALS AND METHODS}

The study was conducted for one year on 40 pregnant females attending our Antenatal Clinic between 28 to 36 weeks of gestation. Esaote Biomedica $3 \mathrm{AU}$ Triplex Doppler machine with convex transducer of 3.5 and $5 \mathrm{MHz}$ frequency was used. The Umbilical Artery (UA), Middle Cerebral Artery (MCA) and Descending Thoracic Aorta (DTA) of the foetus were studied with five indices peak systolic velocity, end diastolic velocity, peak systolic velocity and end diastolic velocity ratio (S/D ratio), Resistive Index (RI) and Pulsatility Index (PI). The foetal weight was estimated sonographically. Those studied at 28 weeks were called for followup study at 32 and 36 weeks. At the end of pregnancy, the foetus was considered as IUGR when the sonographically estimated weight or birth weight was below the $10^{\text {th }}$ percentile of expected weight for a particular gestational period. A comparison of indices was done using unpaired ' $\mathrm{t}$ ' test. The specificity and sensitivity were calculated.

\section{RESULTS}

As the gestational period increases the specificity of RI in UA decreases, whereas the sensitivity of RI increases for UA and MCA and remains the same at all periods for DTA. The highest sensitivity and specificity for RI was seen in the umbilical artery at any period of gestation. The values of sensitivity and specificity of PI and S/D ratio in the prediction of IUGR were highest in the UA at any period of gestation in comparison with MCA and DTA.

\section{CONCLUSION}

The most specific Doppler index for IUGR is S/D ratio followed by PI and RI. The sensitive Doppler index is PI followed by RI and S/D ratio. The UA has better prediction value for IUGR. The sensitivity of Doppler velocimetry of UA goes on increasing and specificity goes on decreasing as pregnancy advances.

\section{KEYWORDS}

Doppler Effect, Foetal Growth Retardation, Sensitivity and Specificity.

HOW TO CITE THIS ARTICLE: Sutagatti JG, Joshi SK. Clinical correlation of Doppler velocimetry and intrauterine growth restriction (IUGR). J. Evolution Med. Dent. Sci. 2017;6(17):1335-1338, DOI: 10.14260/Jemds/2017/290

\section{BACKGROUND}

The growth of the human foetus is a complex process which depends on several factors like genetic makeup of the foetus, nutritional supply to the growing foetus and the environment in which the foetus grows. The nutritional supply and the environment of the foetus are dependent on the function of the foetoplacental circulation. Hence, foetoplacental circulation is one of the important factors which determine the foetal growth. Any abnormality of this circulation results in Intrauterine Growth Restriction (IUGR). IUGR is a foetal growth disorder. It is said to be present in those babies whose birth weight is below the $10^{\text {th }}$ percentile of the average for the gestational age.[1] The foetoplacental circulation can be assessed with the help of Doppler velocimetry.

Financial or Other, Competing Interest: None.

Submission 09-01-2017, Peer Review 12-02-2017,

Acceptance 18-02-2017, Published 27-02-2017.

Corresponding Author:

Dr. Jagadish G. Sutagatti,

Department of Radiodiagnosis,

Karnataka Institute of Medical Sciences (KIMS),

Hubli.

E-mail: drjagadishsutagatti@yahoo.com

DOI: $10.14260 /$ jemds $/ 2017 / 290$
The Doppler effect is explained as the change in frequency and wavelength of a wave, which results from a source moving with respect to the medium, a receiver moving with respect to the medium or even a moving medium. [2] Doppler velocimetry is the only non-invasive method for assessing the foetoplacental haemodynamic status.[3] It enables one to know the umbilical arterial flow/venous flow, the resistance of placenta with the help of Resistive Index (RI) and the velocity/volume of flow in umbilical arteries/veins and other different foetal vessels. Abnormalities of the foetoplacental circulation are forerunners of IUGR. Keeping this in mind, a study was conducted to clinically correlate Doppler velocimetry with IUGR. The primary objectives were to find out the efficacy of Doppler study as a screening test for IUGR and to find the best Doppler index, which could be used routinely for this purpose.

\section{MATERIALS AND METHODS}

The study was conducted on 40 pregnant females in a period of one year at a tertiary medical care hospital. Institutional Ethical Committee approval was obtained prior to the conduct of the study. Written and informed consent was obtained from each patient participating in the study. 
Pregnant women with gestational period between 28 to 36 weeks were selected for the study. These women knew the date of their Last Menstrual Period (LMP), were aged between 18 to 35 years and were attending antenatal clinics of KIMS Hospital, Hubli. The exclusion criteria were patients who did not know the exact LMP, with known LMP but did not turn up for regular Doppler followup in specified period and who turned up for followup but end results of pregnancy were not known (delivered at a place other than our Institute). The selected patient's history and obstetric history were analysed with special emphasis laid on the presence of diabetes mellitus, hypertension, anaemia, nutritional status and habits like smoking and alcoholism. Esaote Biomedica 3 AU Triplex Doppler machine with convex transducer of 3.5 and $5 \mathrm{MHz}$ frequency was used. The recording of images was done with Sony thermal printer. The umbilical artery, middle cerebral artery and thoracic aorta of the foetus were studied with five indices namely peak systolic velocity (S), end diastolic velocity (D), peak systolic velocity and end diastolic velocity ratio (S/D ratio), Resistive Index (RI) and Pulsatility Index (PI). S/D ratio is an index of pulsatility defined as ratio of the maximum (usually peak systolic) to the minimum (usually end diastolic) Doppler shift frequencies. Resistive index is an index of pulsatility defined as the difference between the maximum and minimum Doppler shifts divided by the maximum. Pulsatility index is defined as the difference between the maximum and minimum value divided by the mean value of the wave form over a cycle. The Umbilical Artery (UA) was interrogated at its middle portion where the umbilical cord was freely floating in the liquor. The Middle Cerebral Artery (MCA) was located after identifying the Circle of Willis. The middle cerebral artery was traced up to its portion in the vicinity of the middle cerebral peduncles and interrogated. The descending thoracic aorta was interrogated where it passes through the diaphragmatic hiatus. Three frames of waveforms were obtained for each of the three vessels studied. Inbuilt software was used to give values of the indices after tracing the waveforms. The average of the value was noted in the observation chart. The foetal weight was estimated using head circumference, abdominal circumference and femur length. The pregnant women studied at 28 weeks of gestation were again called for followup of Doppler study at 32 weeks and 36 weeks of gestation. Lastly, at the end of pregnancy, the foetus/baby was considered as IUGR when the sonographically estimated weight or birth weight was below the $10^{\text {th }}$ percentile of expected weight for a particular gestational period. A graph showing correlation of birth weight and gestational age in percentile was used as a reference.[1] Standard Deviation (SD) was calculated for all the mean values of UA, MCA and DTA indices at various gestational periods. A comparison of the indices between normal outcome group patients and abnormal outcome group patients was done using unpaired ' $t$ ' test. Also, the specificity and sensitivity were statistically calculated. The findings of Doppler study and the perinatal outcome were thus analysed and correlated.

\section{RESULTS}

3146 obstetric patients were randomly referred for routine examination. Out of these, 256 cases were referred for obstetric Doppler; 40 of these cases for obstetric Doppler were selected for our study and 216 cases were excluded from the study. The patient's characteristics like age, presence of pregnancy-induced hypertension, diabetes mellitus and presence of cardiovascular/respiratory/other diseases were noted (Table 1). A comparison of UA indices (Table 2), MCA indices (Table 3) and DTA indices (Table 4) between normal outcome group and abnormal outcome group revealed interesting findings. Values for specificity and sensitivity (percentage) of the three indices for the vessels studied were obtained (Table 5). A comparison of specificity and sensitivity between various indices revealed that as the period of gestation increases the sensitivity of RI increases for both umbilical artery and MCA, whereas it remains the same at all periods of gestation for DTA. Also as the period of gestation increases, the specificity of RI in umbilical artery decreases. The highest sensitivity and specificity for RI was seen in the umbilical artery at any period of gestation. The sensitivity and specificity of PI in the prediction of IUGR was maximum in the umbilical artery at any period of gestation in comparison with MCA and DTA. The sensitivity of PI for umbilical artery goes on increasing and specificity goes on decreasing with increasing periods of gestation. The highest values of sensitivity and specificity for S/D ratio in the prediction of IUGR are seen in the umbilical artery. The sensitivity of S/D ratio increases and specificity decreases as pregnancy advances in case of the umbilical artery, whereas it is exactly the reverse in case of DTA.

\begin{tabular}{|c|c|c|c|}
\hline Characteristic & $\begin{array}{c}\text { Normal } \\
\text { Outcome } \\
\text { (n = 24) }\end{array}$ & $\begin{array}{c}\text { Abnormal } \\
\text { Outcome } \\
\text { (n = 16) }\end{array}$ & Total \\
\hline Age & 5 & 4 & 9 \\
\hline$<20$ years & 16 & 10 & 26 \\
\hline 21 - 30 years & 3 & 2 & 5 \\
\hline 31 - 40 years & 0 & 0 & 0 \\
\hline > 40 years & 2 & 13 & 15 \\
\hline $\begin{array}{c}\text { Pregnancy- } \\
\text { induced } \\
\text { hypertension }\end{array}$ & 1 & 0 & 1 \\
\hline Diabetes Mellitus & 21 & 3 & 24 \\
\hline $\begin{array}{c}\text { Clinically } \\
\text { normal } \\
\text { (No other } \\
\text { diseases) }\end{array}$ & \multicolumn{2}{|l}{} \\
\hline \multicolumn{2}{|c|}{ Table } & & \\
\hline
\end{tabular}

Table 1. Patient Characteristics

\begin{tabular}{|c|c|c|c|c|c|}
\hline Indices & $\begin{array}{c}\text { Mean of } \\
\text { Normal } \\
\text { Outcome }\end{array}$ & $\begin{array}{c}\text { Mean of } \\
\text { Abnormal } \\
\text { Outcome }\end{array}$ & $\begin{array}{c}\text { 't' } \\
\text { value }\end{array}$ & $\begin{array}{c}\mathbf{P} \\
\text { value }\end{array}$ & Significance \\
\hline $\mathrm{RI}_{1}$ & 0.68 & 0.79 & 3.23 & 0.01 & $\mathrm{HS}$ \\
\hline $\mathrm{RI}_{2}$ & 0.62 & 0.77 & 4.83 & 0.001 & $\mathrm{VHS}$ \\
\hline $\mathrm{RI}_{3}$ & 0.58 & 0.74 & 12.12 & 0.001 & $\mathrm{VHS}$ \\
\hline $\mathrm{PI}_{1}$ & 1.3 & 2.53 & 3.58 & 0.01 & $\mathrm{HS}$ \\
\hline $\mathrm{PI}_{2}$ & 1.15 & 2.19 & 3.16 & 0.01 & $\mathrm{HS}$ \\
\hline $\mathrm{PI}_{3}$ & 1.03 & 1.48 & 3 & 0.01 & $\mathrm{HS}$ \\
\hline $\mathrm{S} / \mathrm{D}_{1}$ & 2.91 & 7.89 & 2.82 & 0.01 & $\mathrm{HS}$ \\
\hline $\mathrm{S} / \mathrm{D}_{2}$ & 2.74 & 5.76 & 2.77 & 0.05 & $\mathrm{~S}$ \\
\hline $\mathrm{S} / \mathrm{D}_{3}$ & 2.47 & 5.03 & 9.73 & 0.001 & $\mathrm{VHS}$ \\
\hline
\end{tabular}

Table 2. Comparison of Umbilical Artery Indices between Normal Outcome Group vs Abnormal Outcome Group

' $\mathrm{t}$ ' = 2.02 at $\mathrm{p}=0.05 ; \mathrm{DF}=36 ; \mathrm{S}=$ Significant, HS = Highly significant, VHS = Very Highly Significant 


\begin{tabular}{|c|c|c|c|c|c|}
\hline Indices & $\begin{array}{c}\text { Mean of } \\
\text { Normal } \\
\text { Outcome }\end{array}$ & $\begin{array}{c}\text { Mean of } \\
\text { Abnormal } \\
\text { Outcome }\end{array}$ & $\begin{array}{c}\text { ' } \mathbf{t} \text { ' } \\
\text { value }\end{array}$ & $\begin{array}{c}\mathbf{P} \\
\text { value }\end{array}$ & Significance \\
\hline $\mathrm{RI}_{1}$ & 0.793 & 0.78 & 0.60 & 0.05 & $\mathrm{NS}$ \\
\hline $\mathrm{RI}_{2}$ & 0.79 & 0.76 & 0.547 & 0.05 & $\mathrm{NS}$ \\
\hline $\mathrm{RI}_{3}$ & 0.754 & 0.76 & 0.872 & 0.05 & $\mathrm{NS}$ \\
\hline $\mathrm{PI}_{1}$ & 1.89 & 2.03 & 0.437 & 0.05 & $\mathrm{NS}$ \\
\hline $\mathrm{PI}_{2}$ & 1.81 & 1.89 & 0.282 & 0.05 & $\mathrm{NS}$ \\
\hline $\mathrm{PI}_{3}$ & 1.71 & 1.92 & 2.47 & 0.05 & $\mathrm{~S}$ \\
\hline $\mathrm{S} / \mathrm{D}_{1}$ & 5.15 & 5.3 & 0.187 & 0.05 & $\mathrm{NS}$ \\
\hline $\mathrm{S} / \mathrm{D}_{2}$ & 5.35 & 4.79 & 0.671 & 0.05 & $\mathrm{NS}$ \\
\hline $\mathrm{S} / \mathrm{D}_{3}$ & 4.82 & 5.41 & 2.44 & 0.05 & $\mathrm{~S}$ \\
\hline
\end{tabular}

Table 3. Comparison of Middle Cerebral Artery Indices between Normal Outcome Group vs Abnormal Outcome Group

S = Significant NS = Not Significant

\begin{tabular}{|c|c|c|c|c|c|}
\hline Indices & $\begin{array}{c}\text { Mean of } \\
\text { Normal } \\
\text { Outcome }\end{array}$ & $\begin{array}{c}\text { Mean of } \\
\text { Abnormal } \\
\text { Outcome }\end{array}$ & $\begin{array}{c}\text { 't' } \\
\text { value }\end{array}$ & $\begin{array}{c}\mathbf{P} \\
\text { value }\end{array}$ & Significance \\
\hline $\mathrm{RI}_{1}$ & 0.772 & 0.82 & 12.87 & 0.001 & VHS \\
\hline $\mathrm{RI}_{2}$ & 0.788 & 0.83 & 13.17 & 0.001 & $\mathrm{VHS}$ \\
\hline $\mathrm{RI}_{3}$ & 0.793 & 0.826 & 35.64 & 0.001 & VHS \\
\hline $\mathrm{PI}_{1}$ & 2.37 & 2.5 & 0.5271 & 0.05 & NS \\
\hline $\mathrm{PI}_{2}$ & 2.24 & 2.445 & 0.7635 & 0.05 & NS \\
\hline $\mathrm{PI}_{3}$ & 2.21 & 2.629 & 5.337 & 0.01 & $\mathrm{HS}$ \\
\hline $\mathrm{S} / \mathrm{D}_{1}$ & 5.38 & 8.08 & 2.3853 & 0.05 & $\mathrm{~S}$ \\
\hline $\mathrm{S} / \mathrm{D}_{2}$ & 6.02 & 7.908 & 1.502 & 0.05 & NS \\
\hline $\mathrm{S} / \mathrm{D}_{3}$ & 5.89 & 8.578 & 6.686 & 0.001 & $\mathrm{VHS}$ \\
\hline
\end{tabular}

Table 4. Comparison of DTA Indices between Normal Outcome Group vs Abnormal Outcome Group

$\mathrm{S}=$ Significant, NS = Not Significant, VHS = Very Highly Significant

\begin{tabular}{|c|c|c|c|c|c|c|}
\hline \multirow{2}{*}{ Indices } & Umbilical Artery (In \%) & \multicolumn{2}{c|}{ Middle Cerebral Artery (In \%) } & \multicolumn{2}{c|}{ Descending Thoracic Aorta (In \%) } \\
\cline { 2 - 7 } & Specificity & Sensitivity & Specificity & Sensitivity & Specificity & Sensitivity \\
\hline $\mathrm{RI}_{1}$ & 93 & 73 & 53.3 & 39 & 66.6 & 43.4 \\
\hline $\mathrm{RI}_{2}$ & 66 & 78 & 33.3 & 52.1 & 73.33 & 43.4 \\
\hline $\mathrm{RI}_{3}$ & 60 & 82.6 & 42.8 & 60.8 & 64.28 & 33.4 \\
\hline $\mathrm{PI}_{1}$ & 93 & 65.2 & 40 & 52.1 & 73.3 & 34.78 \\
\hline $\mathrm{PI}_{2}$ & 73 & 78 & 13.33 & 73.9 & 66.6 & 34.7 \\
\hline $\mathrm{PI}_{3}$ & 60 & 91.3 & 21.4 & 82.6 & 64.28 & 34.7 \\
\hline $\mathrm{S}_{2} \mathrm{D}_{1}$ & 93.3 & 56.5 & 40 & 52.1 & 80 & 34.7 \\
\hline $\mathrm{S} / \mathrm{D}_{2}$ & 86 & 69 & 33.33 & 60.8 & 86.66 & 30.4 \\
\hline $\mathrm{S} / \mathrm{D}_{3}$ & 80 & 69 & 53.3 & 56.5 & 92.85 & 17.39 \\
\hline \multicolumn{7}{|c|}{ Table 5. Specificity and Sensitivity Comparison between various Indices of Umbilical, } \\
\end{tabular}

\section{DISCUSSION}

We started the study at 28 weeks of gestation, because the umbilical artery usually has end diastolic flow with mature waveforms at 28 weeks and before 28 weeks, absence of end diastolic flow can be a normal phenomenon. We selected the umbilical artery, because it represents the resistance of the placenta; nevertheless the umbilical artery is the most frequently studied vessel in Doppler velocimetry due to its accessibility and the strength of its associations with foetal outcomes.[4] Hypoxia resulting from foetoplacental insufficiency leads to IUGR. Whenever there is hypoxia, the foetal cerebral circulation undergoes autoregulatory changes in the form of cerebral vasodilatation, whereas the foetal peripheral circulation undergoes vasoconstriction. The cerebral vasodilatation and the peripheral vasoconstriction together maintain the foetal cerebral circulation in times of hypoxia. The middle cerebral artery represents the cerebral perfusion of the foetus and the descending thoracic aorta represents the peripheral circulation. Hence, these two vessels were selected for our study. We followed up the three vessels periodically, mainly to monitor known cases of IUGR and for the detection of late onset IUGR. We measured the peak systolic and end diastolic velocities, because they are the basis for all the Doppler indices. The systolic/diastolic velocity ratio is an index which represents the placental resistance in the umbilical artery, the cerebral vascular resistance in the middle cerebral artery and the foetal peripheral resistance in the DTA. The resistive index is a numerical representation of the downstream vascular resistance. The pulsatility index indicates variations in pulsatility and flow over the entire cardiac cycle. Luis A Bracero et al have demonstrated that increased umbilical artery S/D ratio ( $>3$ ) is associated with more perinatal complications like stillbirth, caesarean section for foetal distress and more incidence of admission of babies to intensive care units.[5] Bhushan Lakhkar et al have demonstrated that low diastolic flow and high Doppler indices in the umbilical artery characterise those pregnancies with an abnormal outcome.[6] In our study, cases in the normal outcome group (no IUGR group) showed a mean umbilical artery S/D ratio of $<3$ at 28,32 and 36 weeks of gestation and cases in the abnormal outcome group showed a mean umbilical artery S/D ratio of $>5$ at the same periods. The findings of our study thus support the findings of Bracero et al and Bhushan Lakhkar et al. In a study by Gyawali $\mathrm{M}$ in patients with foetal gestational age 32 weeks or more, it was found that abnormal umbilical artery S/D ratio of 3 or greater for predicting of IUGR gave the sensitivity, specificity, positive predictive value and negative predictive value of $76 \%, 76.9 \%$, $79.2 \%$ and $73.5 \%$ respectively.[7] Our study revealed that the umbilical artery S/D ratio $>3$ at 28 weeks has a sensitivity of $56.5 \%$ and a specificity of $93 \%$ which is highly significant, a sensitivity of $69 \%$ and specificity of $86 \%$ at 32 weeks, a sensitivity of $69 \%$ and specificity of $80 \%$ at 36 weeks of gestation. This clearly suggests that with increasing gestational age, sensitivity increases and specificity decreases. Bhushan Lakhkar et al demonstrated that a high RI characterised those pregnancies with abnormal outcomes including IUGR.[6] Our study supports this finding. We found that an umbilical artery RI of $>0.7$ at 28 weeks of gestation has a sensitivity of $73 \%$ and a specificity of $93 \%$, which is a highly significant finding; at 32 weeks of gestation it has a sensitivity of $78 \%$ and a specificity of $66 \%$; at 36 weeks of gestation it has sensitivity of $82 \%$ and a specificity of $60 \%$. 
This suggests that with advancing pregnancy the sensitivity of RI goes on increasing, whereas the specificity goes on decreasing. Several researchers have shown higher values of PI of umbilical artery in those foetuses, wherein IUGR developed.[6],[8],[9],[10] Our study also confirms the same findings. We found that an umbilical artery PI value of $>1.4$ has a sensitivity of $65 \%$ and a specificity of $93 \%$ at 28 weeks of gestation which is a highly significant finding, a sensitivity of $78 \%$ and a specificity of $73 \%$ at 32 weeks of gestation; a sensitivity of $91 \%$ and a specificity of $60 \%$ at 36 weeks of gestation. This suggests that as the pregnancy advances the sensitivity of umbilical artery PI increases, whereas the specificity falls. Arbeille et al reported that pathological pregnancies with hypertension and IUGR are associated with abnormal middle cerebral artery RI.[11] Dandolo Gramellini et al found that the cerebral to umbilical artery PI ratio proved a better predictor of IUGR.[12] Zha C et al have shown that the middle cerebral artery PI is significantly lower than IUGR foetuses than in normal foetuses.[10] We found higher middle cerebral artery PI in IUGR group. Our findings thus do not confirm the findings of Zha $\mathrm{C}$ et al. Also, according to our study, there is not much of significant difference in middle cerebral RI and S/D ratio values between normal and abnormal outcome groups. Dominic Arduini et al have shown high PI values for the descending thoracic aorta in IUGR foetuses in comparison with normal foetuses.[8] Jan Laurin et al found that blood flow indices in the descending thoracic aorta did not seem to be variables sensitive enough to predict the foetal outcome.[13] In our study, DTA RI index of $>8$ has a sensitivity of $43 \%$ and a specificity of $66 \%$ at 28 weeks of gestation, a sensitivity of $43 \%$ and a specificity of $73 \%$ at 32 weeks of gestation, a sensitivity of $43 \%$ and specificity of $64 \%$ at 36 weeks of gestation. This indicates that the DTA RI has poor sensitivity for IUGR. Also, according to our study, DTA PI and S/D ratio have poor sensitivity for IUGR as evidenced by very low sensitivity values S/D ratio of $17.39 \%$ at 36 weeks of pregnancy and PI of $34.7 \%$ at 28,32 and 36 weeks of pregnancy. However, S/D ratio of $>4$ has a specificity of $80 \%$ at 28 weeks, $86 \%$ at 32 weeks and $92 \%$ at 36 weeks of gestation.

Our study has a few limitations. Our study included arterial vessels. Studies on venous Doppler velocimetry are being conducted nowadays.[3] However, venous Doppler velocimetry has its own limitations.[3] The cerebroplacental ratio, which measures the proportion of flow supplying the brain and placenta is a good parameter for the assessment of IUGR and hypoxia.[14] But we did not measure it. Also, some researchers have shown that there is an ominous association between Absent End-Diastolic Flow (AEDF)/Reversed End Diastolic Flow (REDF) in the umbilical artery and adverse perinatal outcome.[14] However, we did not record these values. Unlike some studies, our study did not deal with any perinatal outcomes other than IUGR.

\section{CONCLUSION}

We conclude on the basis of our study findings that the most specific Doppler index for IUGR is S/D ratio followed by PI and RI. The sensitive Doppler index is PI followed by RI and $\mathrm{S} / \mathrm{D}$ ratio. Amongst the three vessels, the umbilical artery has better prediction value for IUGR. The sensitivity of Doppler velocimetry of umbilical artery goes on increasing and specificity goes on decreasing as the pregnancy advances.

The middle cerebral artery and descending thoracic aorta do not show similar degree of sensitivity and specificity for the prediction of IUGR. Hence, we suggest that Doppler velocimetry of umbilical artery can be used as a screening test in suspected IUGR cases.

\section{REFERENCES}

[1] Dutta DC. Low birth weight baby. In: Konar H, (eds). Textbook of obstetrics including perinatology and contraception. $7^{\text {th }}$ edn. Kolkata: New Central Book Agency 2011:456-67.

[2] Santosh K. Doppler. In: Singh H, Sasana A, Lodha R, (eds). Textbook of Radiology Physics. 1st edn. New Delhi: JP Medical Limited 2016:46-53.

[3] Kaponis A, Harada T, Makrydimas G, et al. The importance of venous Doppler velocimetry for evaluation of intrauterine growth restriction. J Ultrasound Med 2011;30(4):529-45.

[4] Aditya I, Tat V, Sawana A, et al. Use of Doppler velocimetry in diagnosis and prognosis of intrauterine growth restriction (IUGR): a review. J Neonatal Perinatal Med 2016;9(2):117-26.

[5] Bracero LA, Beneck D, Kirshenbaum N, et al. Doppler velocimetry and placental disease. Am J Obstet Gynecol 1989;161(2):388-93.

[6] Lakhkar BN, Ahamed SA. Doppler velocimetry of uterine and umbilical arteries during pregnancy. Indian J Radiol Imaging 1999;9(3):119-25.

[7] Gyawali M, Sharma P, Lalchan S, et al. Prediction of neonatal outcome by umbilical artery velocimetry in intrauterine growth restriction: a study in western Nepal. American Journal of Public Health Research 2015;3(5A):139-43.

[8] Arduini D, Rizzo G, Romanini C, et al. Fetal blood flow velocity waveforms as predictors of growth retardation. Obstet Gynecol 1987;70(1):7-10.

[9] Yoshimura S, Masuzaki H, Gotoh H, et al. The relationship between blood flow redistribution in umbilical artery and middle cerebral artery and fetal growth in intrauterine growth retardation. Nihon Sanka Fujinka Gakkai Zasshi 1995;47(12):1352-8.

[10] Zha C, Li J, Li X. Pulsatility indexes of fetal middle cerebral artery and umbilical artery for predicting intrauterine fetal growth retardation. Zhonghua $\mathrm{Fu}$ Chan Ke Za Zhi 1996;31(6):345-7.

[11] Arbeille P. Fetal arterial Doppler-IUGR and hypoxia. Eur J Obstet Gynecol Reprod Biol 1997;75(1):51-3.

[12] Gramellini D, Folli MC, Raboni S, et al. Cerebralumbilical Doppler ratio as a predictor of adverse perinatal outcome. Obstet Gynecol 1992;79(3):416-20.

[13] Laurin J, Lingman G, Marsál K, et al. Fetal blood flow in pregnancies complicated by intrauterine growth retardation. Obstet Gynecol 1987;69(6):895-902.

[14] Gandhi K, Mehta AV. Role of Doppler velocimetry in growth restricted foetuses. NHL Journal of Medical Sciences 2015;4(1):27-30. 